\title{
Monochromated Electron Energy-Loss Spectroscopy of Interfaces in Beam Sensitive Materials
}

\author{
Jessica A. Alexander ${ }^{1}$ and David W. McComb ${ }^{1}$ \\ 1. Center for Electron Microscopy and Analysis, Department of Materials Science and Engineering, The \\ Ohio State University, Columbus, OH.
}

The practical implementation of electron monochromators in the scanning transmission electron microscope (STEM) in recent years means that spatially resolved electron energy-loss spectroscopy (EELS) can be performed in the STEM with an energy resolution that matches that which can be achieved in synchrotron-based x-ray absorption spectroscopy. The ability to probe chemistry, bonding and electronic properties of materials using monochromated STEM-EELS is already providing new insights in inorganic functional and structural materials. However, realizing the potential of mono-STEM-EELS in the study of organic and biological materials is more challenging.

Organic materials are ubiquitous in all aspects of our daily lives. Increasingly there is a need to understand interactions between different organic phases, or between organic and inorganic materials (hybrid interfaces), in order to gain fundamental knowledge about the origin of their structural and functional properties. Mono-STEM-EELS has the potential to inform the complex structure-property-processing relationships in (and between) these materials. However, low-contrast between phases, and electron beam sensitivity present considerable challenges. To address the latter issue, it is essential to understand the "electron budget" for each experiment; in other words, the electron dose that will lead to changes in the parameter being measured. In many organic and biological materials, this makes study of core-loss excitations prohibitive, in part due to current detector efficiencies. However, the cross-section for valence EELS (VEELS) is considerably higher than core-loss EELS. In this contribution we will discuss our recent efforts to utilize VEELS for the study of such systems.

In most cases VEELS does not give direct information on composition. However, it is well established that the low-loss spectra can be used to extract the real $\left(\varepsilon_{1}\right)$ and imaginary $\left(\varepsilon_{2}\right)$ parts of the complex dielectric function [1]. Since $\varepsilon_{1}$ and $\varepsilon_{2}$ are related to the refractive index $(n)$ and the extinction coefficient $(\kappa)$, both $n$ and $\kappa$ can also be derived over this entire range with high spatial and high energy resolutions. It has always been challenging to obtain accurate data at very low $(<5 \mathrm{eV})$ energy-losses due to the tail of the zero-loss peak (ZLP). Improvements in experimental energy resolution $(\Delta \mathrm{E})$ make it realistic to extract optoelectronic information from organic materials in the energy-loss regions that encompass ultraviolet, visible, and infrared wavelengths.

The acquisition procedures developed to allow collection of Mono-STEM-EELS data from a range of beam sensitive materials will be described. The materials include organic materials commonly used in OPVs, such as $\mathrm{C}_{60}$, copper phthalocyanine ( $\left.\mathrm{CuPc}\right),[6,6]$ phenyl $\mathrm{C}_{61}$ butyric acid methyl ester (PCBM), and poly(3-hexylthiophene) (P3HT), as well as interfaces between them (Figure 1). Recent results on beam sensitive lead-free perovskites for solar cell applications will also be discussed. Finally, the results of investigations of $\mathrm{V}[\mathrm{TCNE}]_{\mathrm{x}}(\mathrm{x}=2$, TCNE: tetracyanoethylene), an organic-based ferromagnetic semiconductor with room temperature magnetic ordering and a bandgap of $0.5 \mathrm{eV}$ will be discussed.

Achieving very high energy resolution presents some challenges for data processing. While the extraction 
of the zero loss peak (ZLP) is often more straightforward compared with non-monochromated spectra, the spectra are recorded at a high dispersion in order to avoid being limited by the point spread function of the system rather than $\Delta \mathrm{E}$. However, in order to extract the optical properties from these spectra it is necessary to have datasets, (a) in which the ZLP is unsaturated and (b) that span an energy range that includes all the valence electron excitations. This necessitates interpolation and splicing of multiple datasets to obtain the full energy-loss spectrum for processing [2,3]. The consequences of these constraints on the use of VEELS for analysis of organic materials will be discussed [4].

\section{References:}

[1] R.F. Egerton, "Electron Energy-Loss Spectroscopy in the Electron Microscope" (Springer, New York).

[2] J.A. Alexander et al, J. Mater. Chem. A 4 (2016), p. 13636.

[3] Jessica A. Alexander et al, Ultramicroscopy 180 (2017), p. 125.

[4] Funding was provided by an AFRL/DAGSI Ohio-Student Faculty Research Fellowship awarded by the Air Force Laboratory Manufacturing and Materials Directorate, and by The Ohio State University through a Distinguished University Fellowship.

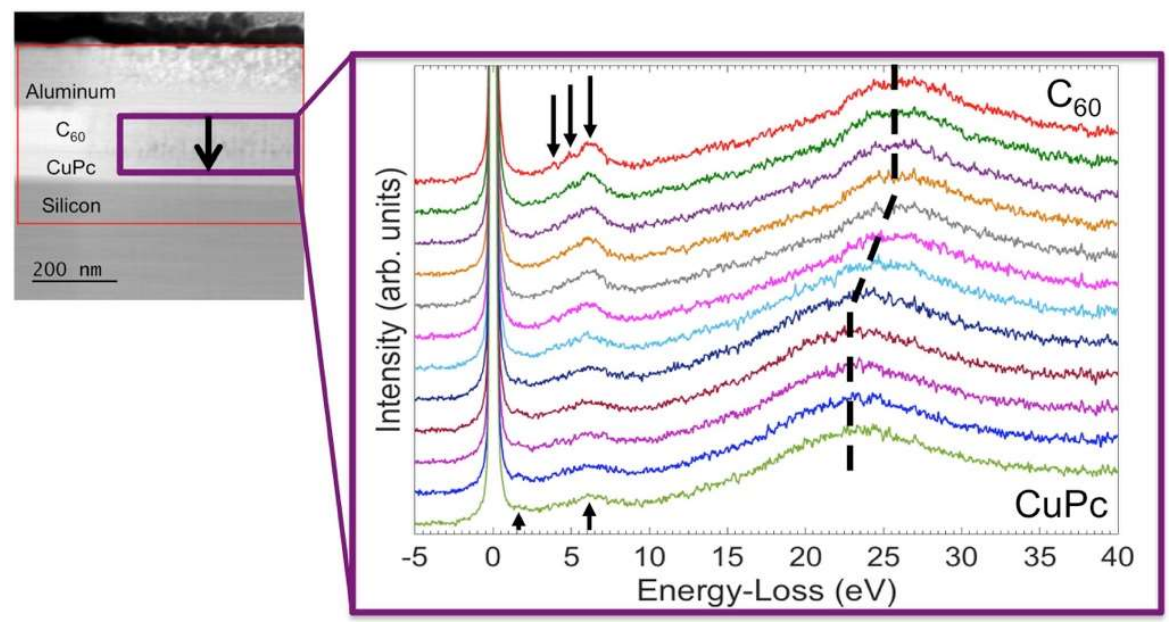

Figure 1. Mono-STEM-EELS data collected across the $\mathrm{C}_{60} / \mathrm{CuPc}$ interface of a bilayer structure. The spectra were collected in $\sim 5 \mathrm{~nm}$ steps. The arrows point out characteristic peaks of the bulk materials, and the dashed line represents the shift in the plasmon peak. 